\title{
The Ca II Infrared Triplet as a stellar activity diagnostic
}

\section{Non-LTE photospheric profiles and definition of the $\boldsymbol{R}_{\mathrm{IRT}}$ indicator}

\author{
V. Andretta ${ }^{1}$, I. Busà ${ }^{2}$, M. T. Gomez ${ }^{1}$, and L. Terranegra ${ }^{1}$ \\ 1 INAF - Osservatorio Astronomico di Capodimonte, salita Moiariello 16, 80131 Napoli, Italy \\ e-mail: andretta@na.astro.it \\ 2 INAF - Osservatorio Astrofisico di Catania, via S. Sofia 78, 95125 Catania, Italy
}

Received 28 July 2004 / Accepted 21 September 2004

\begin{abstract}
This work is part of a larger project on the study of activity in stars of spectral type similar to, or later than the Sun, from PMS to ZAMS, based on the analysis of the high resolution Ca II InfraRed Triplet (Ca II IRT: $\lambda=8498,8542,8662 \AA$ ) observed profiles. Here, a preliminary study on the diagnostic power of these calcium lines has been performed by means of NLTE calculations of the line profiles with an approximate treatment of UV line-blanketing, for a grid of photospheric models with $T_{\text {eff }}=4200,5200,6200 \mathrm{~K}, \log g=4.0,4.5,5.0$ and $[\mathrm{A} / \mathrm{H}]=0.0,-1.0,-2.0$. We used these calculations to estimate the sensitivity of the profiles to changes in stellar parameters and the effect of departures from LTE. As found by other authors, the Ca II triplet NLTE $E Q W$ s (equivalent widths) are quite sensitive to photospheric parameters, in particular to $T_{\text {eff }}$ and [A/H]. On the other hand, we find that the dependence of the Ca II triplet lines central depression $(C D=1$-central relative flux $)$ on $\log g$ and $T_{\text {eff }}$, and to a lesser extent to $[\mathrm{A} / \mathrm{H}]$, is very weak. The departure from LTE is negligible when we consider $E Q W \mathrm{~s}$, unless very metal-poor atmospheres are considered, while $C D$ s can be affected by NLTE by more than $20 \%$. This analysis indicates that in the use of these lines as activity indicators (where the details of the line profile in the core are important), a NLTE treatment is required. Furthermore, we show that a new chromospheric indicator, which we denote $R_{\mathrm{IRT}}$, can be derived from measurements of Ca II IRT line central depressions, provided that rotational broadening is taken into proper account. In order to facilitate the use of the Ca II IRT lines as activity diagnostics, we give interpolation formulae for estimating line $C D \mathrm{~s}$ within the range of stellar parameters of our NLTE calculations.
\end{abstract}

Key words. stars: atmospheres - stars: activity - radiative transfer - line: formation - line: profiles - stars: late-type

\section{Introduction}

The lines at $8498,8542,8662 \AA$, constituting the InfraRed Triplet (IRT) of Ca II, are among the most conspicuous features of the near infrared region of the spectra of G, K, and M stars. Over the last years, with advances in the technology of detectors sensitive to the (near-)IR, the Ca II IRT lines are becoming more and more extensively observed to address a number of topics in different areas. The fields in which the study of the Ca II IRT has found applications span from stellar chromospheres to active galaxies, from starburst regions to globular cluster metallicity analysis. For instance, the Ca II IRT is used for the spectral classification of stars (Munari \& Tomasella 1999) and for the identification of supergiants in the Galaxy (Mantegazza 1992). Or, these lines have a wide application as chromospheric activity indicators (e.g., Dempsey et al. 1993).

A recent summary of the different applications, and an extensive database of low-resolution observations can be found in Cenarro et al. (2001a,b, 2002).

In the context of stellar atmospheres, several authors have tried to model the behavior of the $\mathrm{Ca}$ triplet with basic atmospheric parameters $\left(T_{\text {eff }}, \log g\right.$ and $\left.[\mathrm{A} / \mathrm{H}]\right)$, either using empirical stellar libraries (Mallik 1994, 1997), or using the predictions of stellar atmosphere models. The first attempts to analyse the sensitivity of the Ca II IRT strength to the atmospheric parameters were restricted to the line wings. In this sense, Smith \& Drake (1987, 1990) using Local Thermodynamic Equilibrium (LTE) models concluded that the dependence on metallicity should be larger than that reported by the empirical studies, and that effective temperatures had a non-negligible effect. Erdelyi-Mendes \& Barbuy (1991), also using LTE models, extended the analysis to cooler stars and included the significant contribution of molecular bands. They agreed with Smith \& Drake in that metallicity, rather than gravity, was the main parameter, the $[\mathrm{A} / \mathrm{H}]$ dependence being stronger for lower gravities. Recently, Chmielewski (2000) presented a comprehensive analysis of theoretical LTE modelling of the $\mathrm{Ca}$ lines. His main conclusions about the sensitivity of Ca II IRT line strengths to stellar parameters are that metallicity is a significant parameter in all cases, and that temperature effects can only be neglected for dwarfs between 5000 and $6000 \mathrm{~K}$.

These interesting analyses, based on LTE calculations, do not take into account that the $\mathrm{Ca}$ triplet line cores are 
formed in the stellar chromosphere and that, therefore, nonLTE (NLTE) calculations may be required. Calculations in NLTE of the Ca II IRT equivalent widths, $E Q W$, have been done by Jørgensen et al. (1992). They show that the effects of departures from LTE in the Ca II IRT are negligible since the $E Q W \mathrm{~s}$ are dominated by the photospheric line wings. Obviously those conclusions, valid for $E Q W \mathrm{~s}$, cannot be applied to the core of the line profiles, and thus to the central line depression, $C D$ (if $R$ is the central line flux, normalized to the continuum, $C D=1-R)$. We do in fact expect NLTE effects to be significant in the line core. A proper analysis, in this sense, will be done in this paper, where we will determine the departures from LTE and the dependence on stellar parameters both of the equivalent widths and of line core depths.

In the framework of stellar chromospheric activity, several authors have underscored the diagnostic power of these lines as activity indicators. Linsky et al. (1979) show for example that the radiative loss rates in the $\lambda 8542 \AA$ line are well correlated with radiative loss rates in the $\mathrm{Ca}$ II $\mathrm{H} \& \mathrm{~K}$ and $\mathrm{Mg}$ II $h \& k$ lines. Montes et al. (2000), analysing high resolution spectra of 16 chromospherically active binary systems, find that the Ca II IRT lines turn out to be a very useful chromospheric activity indicator, all the stars analysed showing a clear filledin absorption line profile or even notable emission reversal. Furthermore these authors find that the $E_{8542} / E_{8498}$ ratio is in the range $\approx 1-2$, which is indicative of optically thick emission in plage-like regions, in contrast to the prominence-like material inferred by the $E_{\mathrm{H} \alpha} / E_{\mathrm{H} \beta}$ ratios. These small ratios indicate the existence of distinct sources of Balmer and Ca II IRT emission and suggest that the activity of these very active stars is not simply a scaled-up version of solar activity. Recently, Chmielewski (2000), analyzing the behavior of the central depression $(C D)$ of the $\lambda 8542$ line in a sample of 40 stars, found a correlation between the observed $C D$ and the classical chromospheric indicator $\log R_{\mathrm{HK}}^{\prime}$.

However, the proper subtraction of the photospheric contribution is essential for the correct estimation of the chromospheric contribution to any activity diagnostic. Correction procedures for the photospheric contribution in the cores of the Ca II H\&K lines, for instance, have long been used to derive the $\log R_{\mathrm{HK}}^{\prime}$ indicator (Noyes et al. 1984). To our knowledge, a detailed analysis of such a correction has never been done in a systematic way for the Ca II IRT. Such a correction can be important because, as we will show in this paper, the residual intensity of the Ca II IRT components hardly ever vanishes, even in the absence of a chromosphere, in contrast to what happens to the stronger Ca II H\&K lines.

Moreover, when analyzing high resolution observations, it may be convenient to use the line $C D$ s instead of "residual $E Q W$ s", as discussed in Sect. 3.2. In this case, it is also necessary to take into account the effect of $v \sin \hat{\imath}$ on the line $C D$. This effect has often been assumed to be negligible (as in, e.g., Linsky et al. 1979), or even ignored (as in, e.g., Chmielewski 2000).

In this work we will estimate the corrections to the central depths of the CaII IRT due to these effects, namely: (1) the photospheric contribution; and (2) dependence of CD on $v \sin \hat{\imath}$. These estimates will be accomplished by computing detailed
NLTE photospheric profiles in a grid of model atmospheres (Sects. 2 and 3), broadened over a range of $v \sin \hat{\imath}$ values. The application of such corrections will allow the identification of a pure chromospheric emission indicator, as a function of the basic atmospheric parameters (Sect. 4).

\section{Model calculations and solar test}

In order to investigate the sensitivity of the Ca II IRT line profiles in main-sequence, solar-like stars to basic stellar parameters such as effective temperature, gravity and metallicity, we selected from the NextGen database (Allard \& Hauschildt 1995) a grid of photospheric models with effective temperatures $T_{\text {eff }}=4200,5200$ and $6200 \mathrm{~K}$, gravity $\log g=4.0,4.5,5.0$, and metallicity relative to the $\operatorname{Sun}[\mathrm{A} / \mathrm{H}]=0.0,-1.0,-2.0$.

The coupled equations of radiative transfer and statistical equilibrium were solved for two slightly different calcium atomic models, models M 1 and M 2, using version 2.2 of the code MULTI (Carlsson 1986).

Both $\mathrm{Ca}$ atomic models incorporate eight energy states of CaI, five states of CaII, and the ground state of Ca III. Radiative transfer in nine $b-b$ and thirteen $b-f$ transitions is treated in detail. Collisional data are the same as those used by Drake (1991). The two Ca atomic models differ in the line damping parameters, which are respectively from the Kurucz atomic database (Kurucz \& Bell 1995) (model M 1) and from Smith \& Drake (1990) (model M 2). Moreover, the model containing the Kurucz damping constants also includes collisional data from for the Ca II ${ }^{2} \mathrm{P}_{3 / 2} \leftrightarrow{ }^{2} \mathrm{P}_{1 / 2}$ transition.

The opacity package included in the code takes into account free-free opacity, Rayleigh scattering, and bound-free transitions from hydrogen and metals. The absence of line blanketing in the standard opacity package leads to an overestimate of emerging intensities - especially in the UV - and therefore to an overestimate of photoionization rates, some of which can be important in NLTE calculations.

We have estimated the effect of line blanketing by increasing the background "standard" opacity by a suitable factor, following the method described in Busà et al. (2001).

To illustrate the results of such a procedure, Fig. 1 shows the absolute flux computed using the quiet-Sun atmospheric model C from Fontenla et al. (1993) (henceforth: FAL C model). The three solid lines refer to the upper-limit, average and lower-limit of the observed solar flux given by Vernazza et al. (1976).

Finally, we adopted the value for solar calcium abundance given by Chmielewski (2000): $\log \epsilon_{\odot}(\mathrm{Ca})=6.36$, which is fully consistent with the standard value given by Grevesse \& Sauval (1998).

We tested our procedure and data set on the Sun using again the FAL $\mathrm{C}$ atmospheric model, which, we recall, is a semi-empirical model of the solar atmosphere, encompassing both the photosphere and the chromosphere. Figure 2 shows the synthetic Ca II IRT line profiles obtained using the M 1 and M 2 model atoms (dashed and dotted lines, respectively), compared with the Kitt Peak solar flux atlas (Kurucz et al. 1984). In the computation of the model grid we used the M 1 model atom (the model atom using data from Kurucz \& Bell 1995) which 


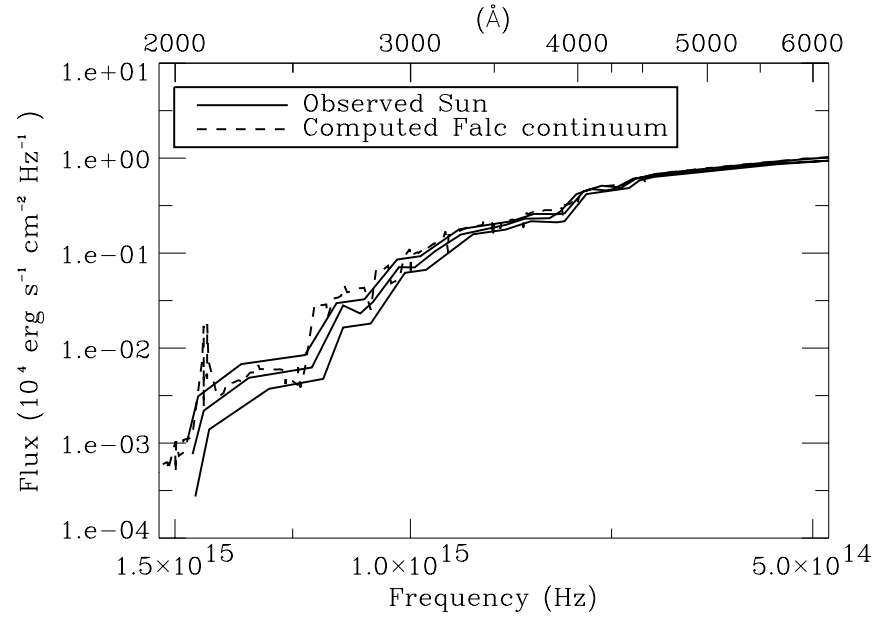

Fig. 1. Comparison between the observed solar fluxes from Vernazza et al. (1976) (solid line) and the FAL C computed continuum.

seems to reproduce the solar observation better. The discrepancy between $C D$ s computed from synthetic spectra and those derived from the observed solar lines is of about $0.3 \%, 3.8 \%$, and $2.2 \%$, for the $\lambda 8498, \lambda 8542$, and $\lambda 8662$ components, respectively. These values can be taken as an estimate of the uncertainty on the synthetic profiles due to the uncertainty of the atomic model parameters.

\section{NLTE effects and sensitivity to stellar parameters}

NLTE effects and sensitivity of the Ca II IRT lines to the stellar parameters temperature, gravity and metallicity have been investigated by analysing the profiles obtained by the NLTE line-blanketed computations from the adopted grid of models. Since the behaviour of the three lines is quite similar, here we show only the $\lambda 8662$ line profiles; however, the correlations of $E Q W$ s and $C D$ with stellar parameters are given for all three lines.

\subsection{NLTE effects}

The effects of NLTE on the Ca II IRT EQWs have been calculated for the 27 photospheric models of the selected grid. The result of such a calculation for the $\lambda 8662$ line is shown in Fig. 3. In that figure, crosses indicate solar metallicity models, triangles refer to $[\mathrm{A} / \mathrm{H}]=-1.0$ and squares to $[\mathrm{A} / \mathrm{H}]=-2.0$; increasing symbol size denotes increasing $\log g$; likewise, increasing symbol thickness corresponds to increasing $T_{\text {eff }}$.

We find that the effect of departure from LTE on the $E Q W$ s is negligible (less than $10 \%$ ), except for the models with lowest metallicity, for which we can have variations of up to $30 \%$. Furthermore, we notice that NLTE effects are stronger in lower $T_{\text {eff }}$ and lower $\log g$ atmospheres.

The limited effect of NLTE on the EQWs of the Ca II IRT can be understood by noting that in the range of stellar atmospheric parameters we have considered, the most significant contribution to the $E Q W$ s of these lines comes from the wings, which are formed deep in the photosphere, where the source
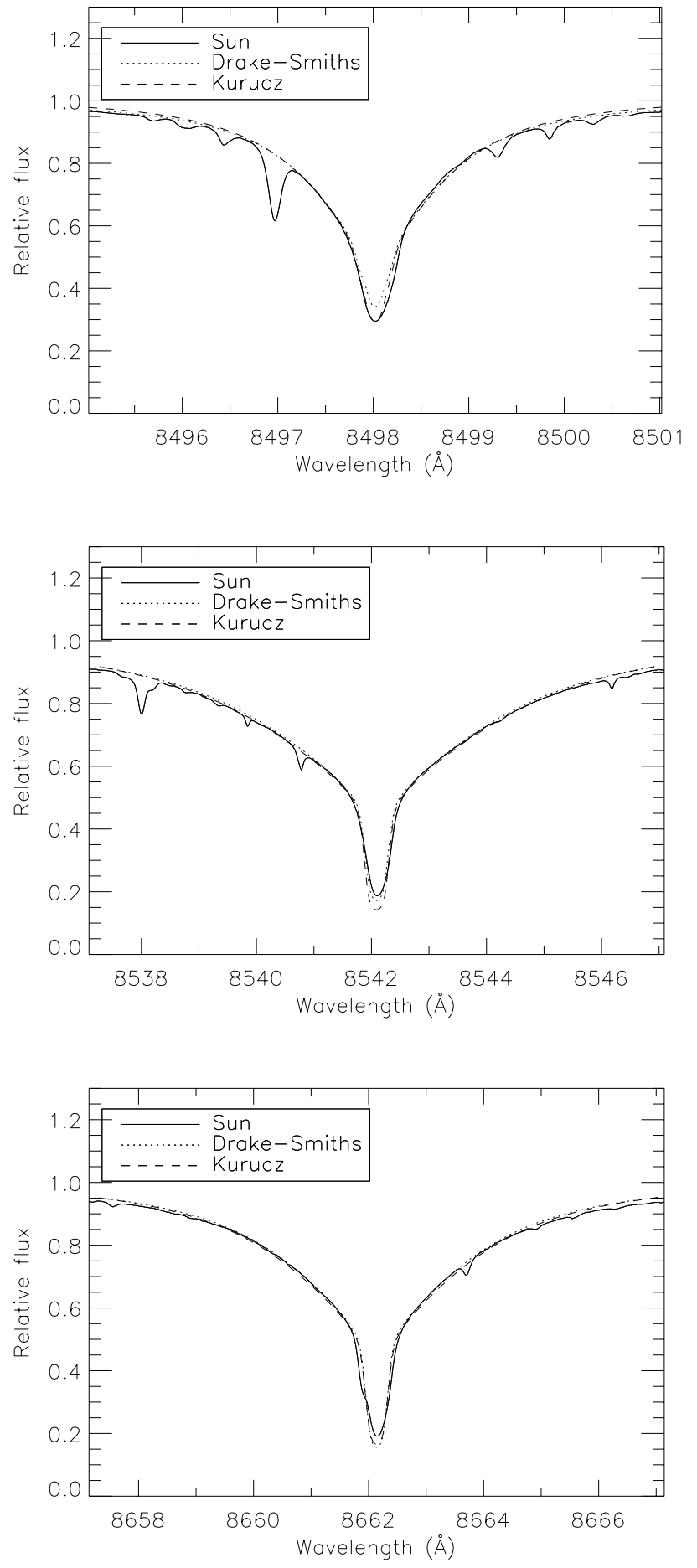

Fig. 2. Synthetic Ca II IRT lines obtained from the FAL C model of the quiet Sun, using the damping constants from Smith \& Drake (1990) (dotted line) and using the damping constants from the Kurucz database (dashed line) compared with a solar flux atlas (thick line).

function is normally close to LTE. Our results are therefore in agreement with the findings of Jørgensen et al. (1992).

NLTE effects on the Ca II IRT line profiles are highlighted in Fig. 4, which shows a comparison between the computed LTE and NLTE profiles obtained for two photospheric models with $T_{\text {eff }}=4200 \mathrm{~K}$ and $\log g=4.0:[\mathrm{A} / \mathrm{H}]=0.0$ (lower panel; NextGen model 42-4.0-0.0) and $[\mathrm{A} / \mathrm{H}]=-2.0$ (upper panel; 


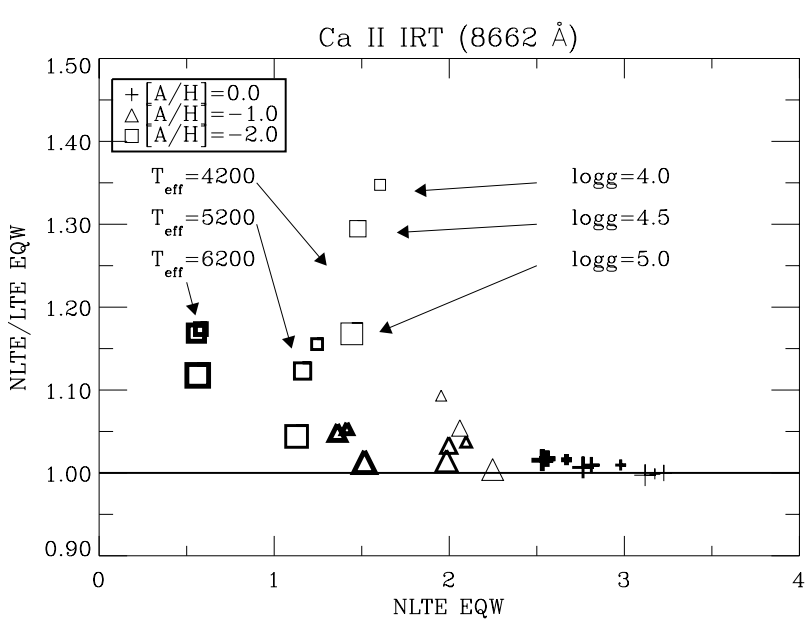

Fig. 3. EQWs departure from LTE for the $\lambda 8662 \mathrm{Ca}$ II lines.
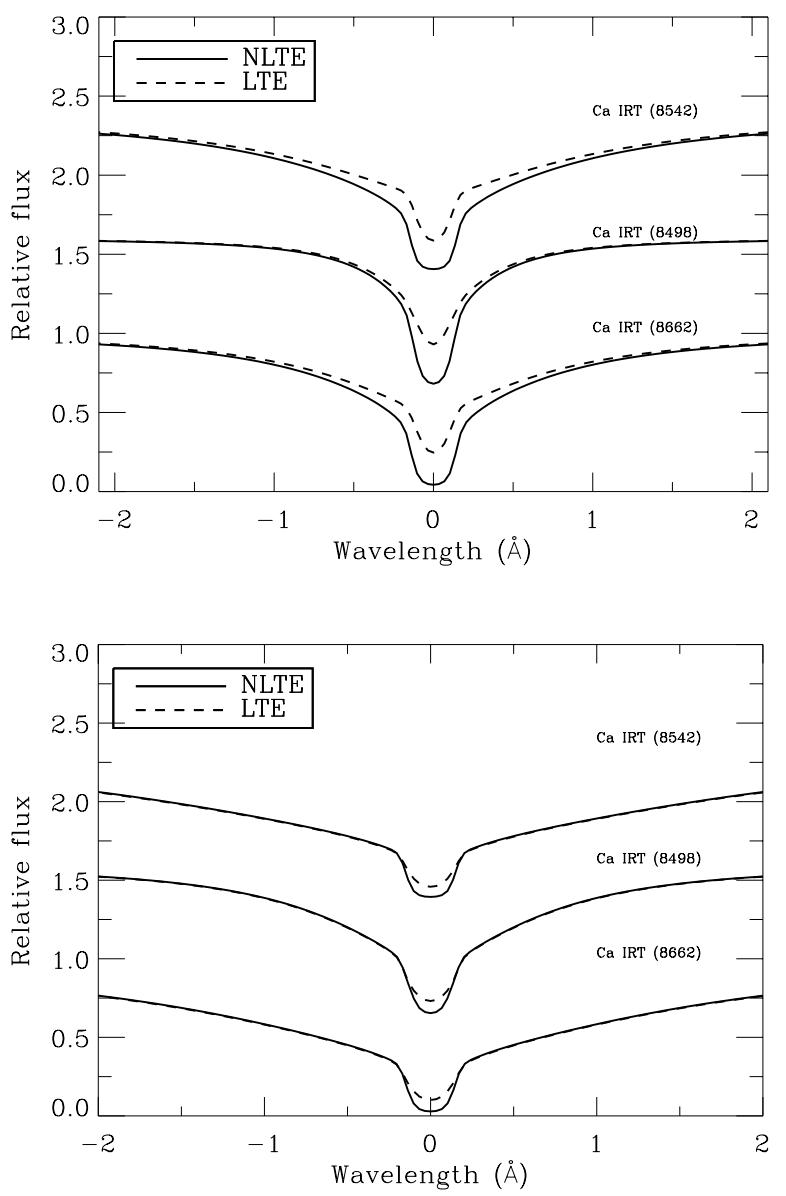

Fig. 4. Ca II IRT line profiles obtained for the NextGen models 42-4.0-0.0 (lower panel) and 42-4.0-2.0 (upper panel) in LTE (dashed line) and NLTE (solid line) approximations.

NextGen model 42-4.0-2.0). We have chosen these two models because of the different effect NLTE has on the line profiles: as shown in Fig. 3, the higher-metallicity model is much less affected than the low-metallicity one. These figures show that NLTE affects the Ca II IRT CDs mainly in metal-poor stars, as hinted at by the behaviour of the EQWs. However, it should be noted that even in the models least affected by NLTE, while the

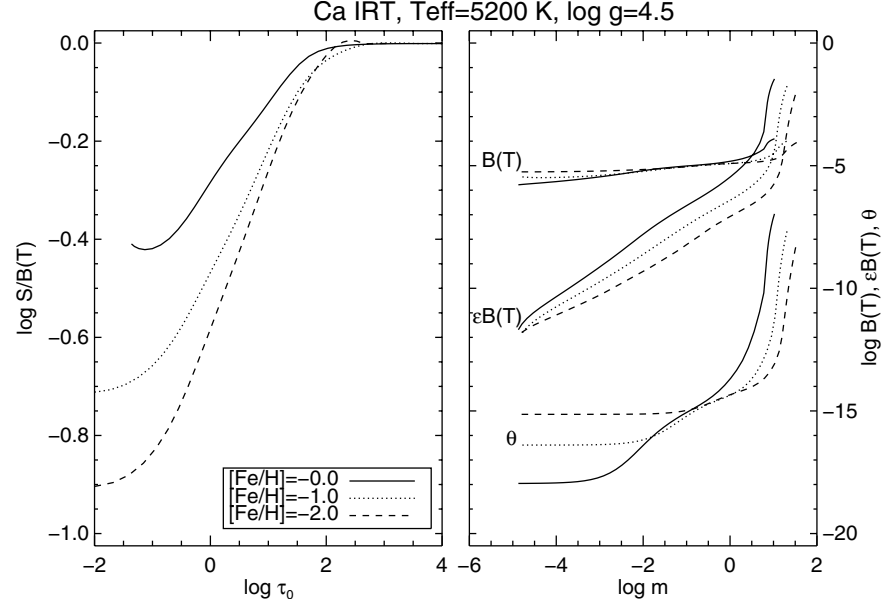

Fig. 5. Dependence of the source function of the Ca II IRT on central optical depth and metallicity (left panel), for atmospheres with $T_{\text {eff }}=5200 \mathrm{~K}$ and $\log g=4.5$. The dependence with depth in the atmosphere (column mass scale) of the coupling terms in the source function, $\epsilon B_{v}(T)$ and $\theta$, is shown in the right-hand panel, together with the LTE source function, $B_{v}(T)$.

effect on the $E Q W \mathrm{~s}$ is less than $6 \%$, the $C D$ s can differ by more than $20 \%$. Since it is the line central depression of the Ca II IRT which is better correlated with stellar chromospheric activity (Mallik 1997), an NLTE treatment of these lines is required if we are to investigate their potential as activity indicators.

The stronger departures from LTE in the lower-metallicity profiles can be due to either to the higher UV flux - less line blanketing - or to the lower electron density - fewer donors - of those atmospheres. The former effect would increase the photoionization rates; the latter would decrease the coupling with the electron thermal pool. Both effects tend to strengthen departure from LTE.

To illustrate this point, we examine the source function of the multiplet, considered as a single line:

$S_{v}=\frac{2 h \bar{v}^{3}}{c^{2}}\left(\frac{g_{l} N_{u}}{g_{u} N_{l}-g_{l} N_{u}}\right)$

where $\bar{v}$ is the average frequency of the transition, and $N_{l}$ and $N_{u}$ are the populations of the lower and upper levels, respectively: $3 \mathrm{p}^{6} 3 \mathrm{~d}^{2} \mathrm{D}^{\mathrm{e}}$ and $3 \mathrm{p}^{6} 4 \mathrm{p}^{2} \mathrm{P}^{\circ}$, while $g$ denotes their statistical weights. In writing the above expression, we neglect the differences in line profiles between the three components of the multiplet, as well as the fact that the fine structure levels are only approximately populated according to their statistical weights, due to NLTE effects.

Figure 5 shows the drop of the source function - normalized to the LTE source function, $B_{v}(T)$ - with optical depth, typical of resonant scattering. The surface value of the normalized source function - related to the central flux in the emerging profile - is driven essentially by the coupling terms in the source function. In a two-level atom without continuum, the coupling is determined by the collisional terms between the two levels, $\epsilon B_{v}(T)$, where $\epsilon=C_{u l}[1-\exp (-h v / k T)] / A_{u l}: C_{u l}$ is the collisional de-excitation rate, and $A_{u l}$ is the Einstein coefficient for spontaneous de-excitation. In this regard, we recall the textbook result valid in a semi-infinite atmosphere with constant 

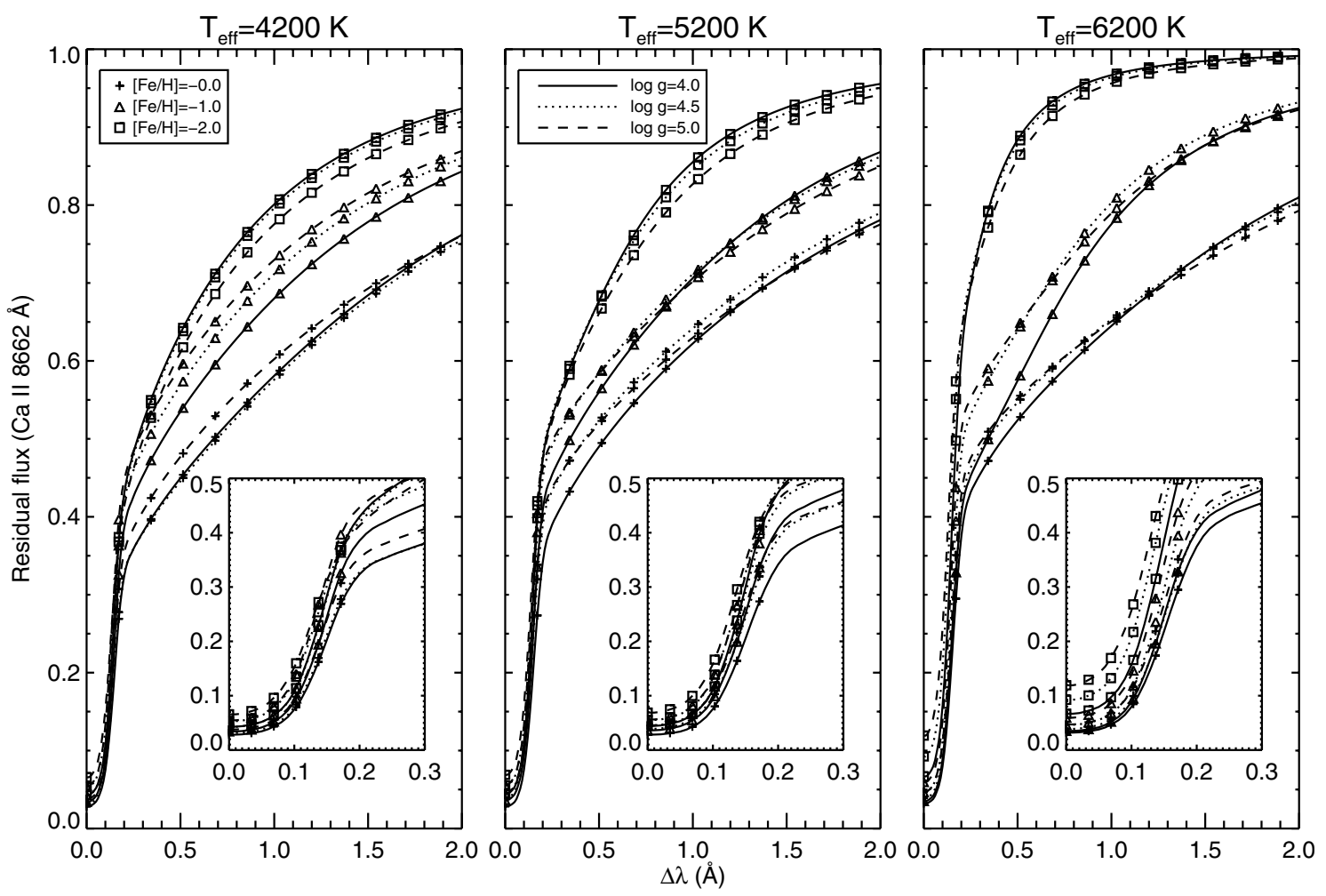

Fig. 6. Ca II $8662 \AA$ A line profiles for the whole grid of models. Crosses indicate the solar metallicity models, triangles refers to $[\mathrm{A} / \mathrm{H}]=-1.0$ and squares to $[\mathrm{A} / \mathrm{H}]=-2.0$; solid lines indicate $\log g=4.0$ models, dotted lines $\log g=4.5$ and dashed $\operatorname{lines} \log g=5.0$ models. Details of line cores are shown in the close-up plots.

properties: $S(\tau=0)=\sqrt{\epsilon /(1+\epsilon)} B \approx \sqrt{\epsilon} B$, if $\epsilon \ll 1$ (e.g., Mihalas 1978, where however $\epsilon$ is denoted by $\epsilon^{\prime}$ ). If the interaction with the photoionization continuum is also taken into account, an additional coupling parameter is introduced in the source function, set by the photoionization and recombination rates, that we denote by $\theta$, again following Mihalas (1978). If $k$ denotes the continuum, and $P$ the total transition rates (sum of radiative plus collisional), in a two-level atom plus continuum we have $\theta=2 h \bar{v}^{3} / c^{2}\left[g_{l} P_{l k} P_{k u} /\left(P_{k l}+P_{k u}\right)\right] /\left(g_{u} A_{u l}\right)$.

In our case, these two coupling terms already give a good qualitative insight into the NLTE processes affecting the source function. The run of these terms with depth in the atmosphere is shown in the right-hand panel of Fig. 5. The decrease with metallicity of the photoionization term, $\theta$, in the upper atmospheric layers, reflects the lower UV flux due to higher line blocking, while the increase of the collisional term, $\epsilon B_{v}(T)$, follows the higher electron density of more metal-rich photospheres.

From Fig. 5, it is clear that the collisional term dominates over the photoionization term. These terms, shown in the righthand panel, completely characterize the line source function only in a three-level atom (two bound levels plus continuum), so they can only approximately characterize the multi-level case shown in the left-hand panel. Moreover, it is also dangerous to apply a simple scaling law of the type $S(\tau=0) / B \propto \sqrt{\epsilon}$ to atmospheres such as the ones we are considering, with strong gradients of the relevant quantities (temperature, density, radiation field). Nevertheless, it is tempting to note that indeed the normalized source function of the Ca II IRT lines follows the decrease of the collisional term, rather than the increase of the photoionization coupling. It should also be remarked that, while $S / B$ increases with metallicity, $S$ in fact decreases, and thus the line becomes deeper. This is because the effect of metallicity in NextGen models on the surface temperature, and consequently on the value of Planck's $B(T)$, is opposite, and of larger magnitude (see again right-hand panel of Fig. 5).

In short, in the grid of stellar models we have considered, NLTE effects in the Ca II IRT are stronger in lower metallicity photospheres because of their lower electron densities. For the same reason, NLTE is more important in the lower gravity models, since the electron density, along with the overall pressure, is also reduced in those atmospheres. Incidentally, on the basis of these fairly simple considerations, we may extrapolate that NLTE effects in the CaII IRT lines should be even more important in the atmospheres of cool giants, possibly not just in the core as in these main-sequence stars, but over the entire line profile.

Aside from the above qualitative considerations, a more refined analysis of NLTE effects on these purely photospheric profiles is not of much interest: in most, if not all realistic cases, the presence of a chromosphere would fundamentally alter the mechanisms of NLTE line formation.

\subsection{Dependence on stellar parameters}

Figure 6 shows the behaviour of the CaII IRT NLTE $\lambda 8662$ profiles with changes in the metallicity and $\log g$ in main-sequence stars with $T_{\text {eff }}=4200 \mathrm{~K}$ (left-hand panel), 


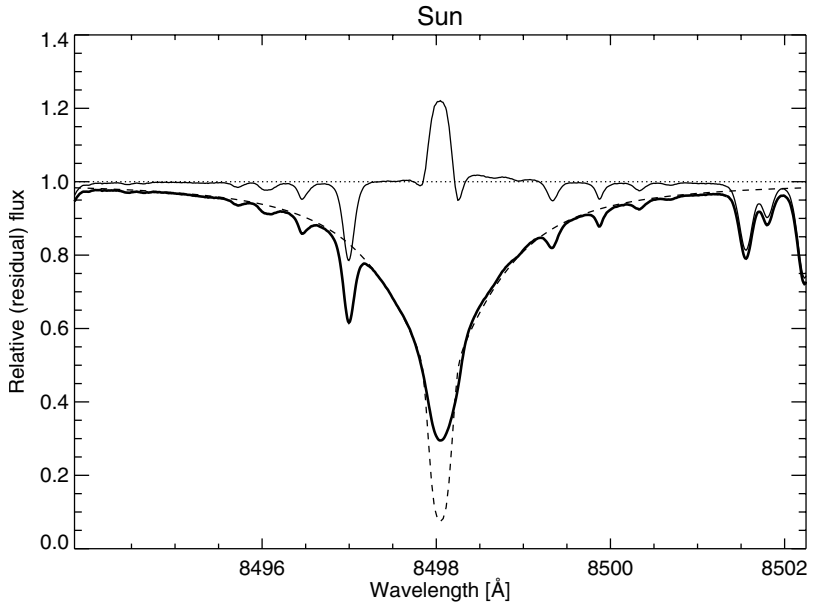

Fig. 7. Solar profile of the $\lambda 8498$ line (solid, thick line), compared to the synthetic photospheric profile of a star with $T_{\text {eff }}=5780 \mathrm{~K}$ and $\log g=4.44$ (dashed line). The residual profile (solid, thin line) is also shown.

$T_{\text {eff }}=5200 \mathrm{~K}$ (middle panel) and $T_{\text {eff }}=6200 \mathrm{~K}$ (right-hand panel). Crosses indicate solar metallicity models, triangles refers to $[\mathrm{A} / \mathrm{H}]=-1.0$, and squares to $[\mathrm{A} / \mathrm{H}]=-2.0$; solid lines indicate $\log g=4.0$ models, dotted lines $\log g=4.5$, and dashed lines $\log g=5.0$ models.

Since the profiles are dominated by the LTE broad wings, for discussion on the dependence of the EQWs on stellar parameters we refer the reader to the existing literature (e.g., Chmielewski 2000, and references therein). We only remark here how the shape of the profiles appear to be only mildly dependent on $\log g$.

On the other hand, the $C D$ s are nearly constant: for the photospheres in our grid, strong resonant scattering leads to small residual fluxes in the core. Only in hotter, more metal-deficient models is the line core at $10 \%$ level of the continuum intensity.

However, even if small, the residual flux in the core of the line is still not negligible, and thus needs to be taken into account in comparisons with observations.

A common approach to estimate the chromospheric contribution in the core of the CaII IRT lines typically involves the subtraction of the profile of a slowly rotating star, broadened by the proper rotational profile of the object star. Such a procedure assumes that the template star is completely chromospherically inactive. Quite often, however, not enough attention is paid to the possibility that template stars may in effect be somewhat active. An example of such an approach is given by Soderblom et al. (1993), who use the Sun as a template. To illustrate this point, in Fig. 7 we compute the "photospheric" profile of the $\lambda 8498$ line, for a star with $T_{\text {eff }}=5780 \mathrm{~K}$ and $\log g=4.44$, by means of a bilinear interpolation in $T_{\text {eff }}$ and $\log g$ of our computed profiles, at each wavelength point. We show such a profile, compared with the actual solar profile shown already in Fig. 2, together with its residual. Clearly, using the Sun as "template" star leads to a systematic underestimation of the chromospheric emission in solar-type stars. In fact, we feel it would be very hard to find a really inactive star against to which measure emission in lines such as the Ca II IRT. It may even be argued that there is little reason to expect any solar-like star, unless very old and non-convective, to be completely chromospherically inactive.

An alternative approach might involve subtracting computed photospheric profiles from observed lines, analogously to what is shown in Fig. 7. We plan to apply such a procedure in the follow-up papers of this series.

On the other hand, a different approach could be adopted in the case of observations with sufficiently high resolution $(R>50000)$, so that it is not necessary to take into account instrumental broadening. In that case, it is advantageous to use the line $C D$ as a proxy for chromospheric activity. This approach has been employed, for example, by Chmielewski (2000) as well as by others (see again Chmielewski 2000, and references therein).

\subsubsection{Interpolation over the stellar grid}

To facilitate the comparison of our computed $C D$ s with observations, we provide here interpolation formulae valid within the range of stellar photospheric parameters of our grid.

For completeness, we also provide interpolation formulae for $E Q W \mathrm{~s}$. We note that, in principle, our computed $E Q W \mathrm{~s}$ could also be subtracted from $E Q W \mathrm{~s}$ measured from observed profiles, to obtain residual $E Q W \mathrm{~s}$. Of course, since the profiles of the Ca II IRT lines in our grid are largely dominated by their photospheric wings, the errors inherent in such a procedure would be large.

In our interpolation formulae, we choose to normalize the stellar parameters upon which the $E Q W \mathrm{~s}$ and $C D$ s depend, as follows:

$$
\begin{aligned}
x & \equiv\left(T_{\mathrm{eff}}-5780 \mathrm{~K}\right) /(1000 \mathrm{~K}) \\
y & \equiv 2 \times(\log g-4.44) \\
z & \equiv[\mathrm{A} / \mathrm{H}] .
\end{aligned}
$$

These non-dimensional quantities span the range of 2 units over the adopted grid, and have the value of zero for the Sun.

The adopted expression for the interpolation formulae is based on a power-series expansion of the unknown function $c(x, y, z)$ (where $c$ can be either $E Q W$ or $C D$ ) truncated to the second-order:

$$
\begin{aligned}
c(x, y, z) \approx & c_{0}+c_{x} x+c_{y} y+c_{z} z \\
& +c_{x x} x^{2}+c_{y y} y^{2}+c_{z z} z^{2} \\
& +c_{x y} x y+c_{x z} x z+c_{y z} y z .
\end{aligned}
$$

In Table 1 we list the best-fit interpolation coefficients for our computed NLTE EQWs, for each Ca II IRT component. The fit produced by Eq. (2) with the coefficients of Table 1 for this line are within a few percent from the actual computed values (with a standard deviation of $4.7 \%$ and a maximum residual of $12.3 \%$ ). The interpolated values to the $E Q W \mathrm{~s}$ of the other two components have similar residuals.

The stronger influence of metallicity on $E Q W$ s for the three lines, already noted from Fig. 6, is also evident from the relative strength of the linear coefficients of Table 1.

The best-fit coefficients for the NLTE CDs are instead given in Table 2. In this case, residuals are at most of 1.6\% (standard 
Table 1. Interpolation coefficients for the Ca II IRT $E Q W$ s. These coefficients have been computed for $E Q W s$ measured in $\AA$. From the definition of the interpolation function and of the independent variables (see text), the coefficients $c_{0}$ correspond to the $E Q W \mathrm{~s}$ of a star with the same atmospheric parameters as the Sun.

\begin{tabular}{rcccccccccc}
\hline \hline Line $\backslash$ Coeff.: & $c_{0}$ & \multicolumn{1}{c}{$c_{x}$} & \multicolumn{1}{c}{$c_{y}$} & \multicolumn{1}{c}{$c_{z}$} & \multicolumn{1}{c}{$c_{x x}$} & \multicolumn{1}{c}{$c_{y y}$} & \multicolumn{1}{c}{$c_{z z}$} & \multicolumn{1}{c}{$c_{x y}$} & \multicolumn{1}{c}{$c_{x z}$} & $c_{y z}$ \\
\hline $8498 \AA:$ & 1.31 & -0.200 & 0.0192 & 0.563 & -0.0593 & 0.0139 & 0.0621 & 0.00524 & 0.0356 & 0.0108 \\
$8542 \AA:$ & 3.52 & -0.550 & 0.0404 & 1.55 & -0.167 & 0.0438 & 0.161 & 0.0159 & 0.120 & 0.0102 \\
$8662 \AA:$ & 2.73 & -0.423 & 0.0313 & 1.20 & -0.126 & 0.0331 & 0.129 & 0.0111 & 0.0890 & 0.00938 \\
\hline
\end{tabular}

Table 2. Interpolation coefficients for the Ca II IRT CDs. These coefficients have been computed for $C D$ s measured in percent of the continuum intensity.

\begin{tabular}{rcccccccccc}
\hline \hline Line $\backslash$ Coeff.: & \multicolumn{1}{c}{$c_{0}$} & \multicolumn{1}{c}{$c_{x}$} & \multicolumn{1}{c}{$c_{y}$} & \multicolumn{1}{c}{$c_{z}$} & \multicolumn{1}{c}{$c_{x x}$} & \multicolumn{1}{c}{$c_{y y}$} & $c_{z z}$ & $c_{x y}$ & $c_{x z}$ & $c_{y z}$ \\
\hline $8498 \AA:$ & 93.5 & -1.27 & -1.45 & 1.39 & -0.951 & -0.118 & -1.15 & -0.601 & 1.47 & 0.884 \\
$8542 \AA$. & 97.2 & -0.455 & -0.756 & 0.778 & -0.429 & -0.0553 & -0.479 & -0.291 & 0.728 & 0.360 \\
$8662 \AA$. & 96.7 & -0.555 & -0.831 & 0.684 & -0.498 & -0.0770 & -0.639 & -0.325 & 0.799 & 0.482 \\
\hline
\end{tabular}

Table 3. Interpolation coefficients, $a$ and $b$, for $\Delta C D_{\text {conv }} \equiv C D_{\text {conv }}(v \sin \hat{\imath})-C D$, as given in Eq. (3). As in Table 2, these coefficients have been computed for $C D$ s measured in percent of the continuum intensity.

\begin{tabular}{rcccccccccc}
\hline \hline Line $\backslash$ Coeff.: & $a_{0}$ & $a_{x}$ & $a_{y}$ & $a_{z}$ & $a_{x x}$ & $a_{y y}$ & $a_{z z}$ & $a_{x y}$ & $a_{x z}$ & $a_{y z}$ \\
\hline $8498 \AA:$ & -11.6 & -1.04 & -0.840 & 2.65 & -0.524 & 0.263 & -0.282 & 0.0296 & 0.655 & -0.325 \\
$8542 \AA:$ & -7.90 & -0.831 & -0.883 & 1.85 & -0.393 & 0.170 & -0.115 & -0.196 & 0.718 & -0.103 \\
$8662 \AA:$ & -9.01 & -0.917 & -0.902 & 2.04 & -0.444 & 0.183 & -0.189 & -0.171 & 0.779 & -0.136 \\
\hline
\end{tabular}

deviation: $0.6 \%$ ), with comparable values for the $8542 \AA$ component, and somewhat larger (maximum residual: $3.2 \%$; standard deviation: $1.3 \%$ ) for the $8498 \AA$ line.

We note that the second-order interpolation formulae employed here give somewhat more accurate results for $C D$ s compared to $E Q W \mathrm{~s}$. The main reason lies in the fact that the overall shape of the line profiles changes significantly with stellar parameters (see Fig. 6), thus introducing "higher-frequency" variations in the $E Q W \mathrm{~s}$. More accurate results could probably be obtained adopting a finer grid in stellar parameters together with higher order interpolation functions.

\subsubsection{The effect of rotational broadening}

Over the grid of stellar parameters we have considered, the range of variation of the CaII IRT $C D$ s is rather narrow: from 88 to $98 \%$. Thus, it is reasonable to expect that line broadening by stellar rotation could contribute to the actual observed values of the $C D$.

To quantify this effect, we have broadened the calculated profiles with values of $v \sin \hat{\imath}$ spanning the range $v \sin \hat{\imath}<$ $20 \mathrm{~km} \mathrm{~s}^{-1}$ (typical values for low-activity stars), with steps of $1 \mathrm{~km} \mathrm{~s}^{-1}$. We found that indeed the effect is not negligible, as shown below.

We found that, over such a range of $v \sin \hat{\imath}$ values, it is possible to accurately fit the $C D$ s calculated from the convolved profiles, $C D_{\text {conv }}$, with the following expression:

$$
\begin{aligned}
C D_{\mathrm{conv}}= & C D(v \sin \hat{\imath}=0) \\
& +a(x, y, z) \times \log _{10}\left[1+\left(v \sin \hat{\imath} / v_{b}\right)^{\alpha}\right],
\end{aligned}
$$

where $v_{b}=5 \mathrm{~km} \mathrm{~s}^{-1}$, and $\alpha=5$. The value $v_{b}$ approximately corresponds to the half-width of the line cores in our photospheric profiles. Consequently, the variation of $C D_{\text {conv }}$ with $v \sin \hat{\imath}$ is rather small for $v \sin \hat{\imath}<v_{b}$. This behaviour is reproduced by the fit function given in Eq. (3).

To account for the variation of coefficient $a(x, y, z)$ with stellar parameters, we employ the same interpolation formula as for the $E Q W \mathrm{~s}$ and $C D \mathrm{~s}(v \sin \hat{\imath}=0)$, given by Eq. (2). The corresponding interpolation coefficients for $a(x, y, z)$ are given in Table 3. The standard deviations of the distribution of errors introduced by using the interpolation function of Eq. (2) to approximate the values $C D(v \sin \hat{\imath}=0)$ and $a(x, y, z)$ of Eq. (3), amount to $4.9 \%, 2.1 \%$, and $2.4 \%$ for the $\lambda 8498,8542$, and 8662 lines, respectively. In the range $v \sin \hat{\imath}<20 \mathrm{~km} \mathrm{~s}^{-1}$, the errors for the two components at 8542 and $8662 \AA$ are always below $10 \%$, while being as large as $20 \%$ for the $\lambda 8492$ line, with the largest errors occurring in the lowest metallicity models. In the range $v \sin \hat{\imath}<15 \mathrm{~km} \mathrm{~s}^{-1}$, however, the largest errors amount to $14 \%, 5.2 \%$, and $6.6 \%$ for the $\lambda 8498,8542$, and 8662 lines, respectively.

\section{Central line depression: An activity indicator?}

Chmielewski (2000) analysed the behavior of the $C D(\lambda 8542)$ line in a sample of 40 stars. The author finds a correlation between the observed $C D$ and the pure chromospheric indicator $\log R_{\mathrm{HK}}^{\prime}$. However, as the author stresses, the observed $C D$ cannot be considered a pure chromospheric indicator because no correction for the photospheric contribution is made. Moreover, as we have shown in Sect. 3.2.2, the effect of $v \sin \hat{\imath}$ on line $C D$ s is not negligible; this fact, compounded with the 


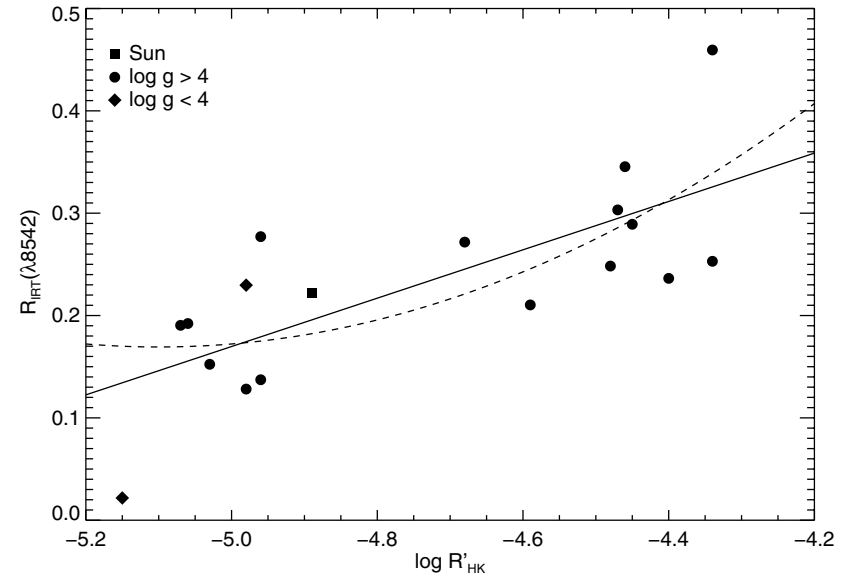

Fig. 8. $R_{\mathrm{IRT}}$ (component at $8542 \AA$ ) versus $\log R_{\mathrm{HK}}^{\prime}$ for those stars with known $v \sin \hat{\imath}$ listed by Chmielewski (2000).

well-known correlation in late-type stars between activity and rotation rates, must be taken into account when attempting any use of the Ca II IRT for studying stellar activity.

We therefore suggest as a better estimator of the chromospheric contribution to the CaII IRT, for a star of known $T_{\text {eff }}$, $\log g,[\mathrm{~A} / \mathrm{H}]$ and $v \sin \hat{\imath}$ the use of a new index: $R_{\mathrm{IRT}}$. This index is defined as the the difference between the observed $C D$ and computed, $v \sin \hat{\imath}$-convolved $C D_{\text {conv }}(\mathrm{NLTE})$ :

$R_{\mathrm{IRT}} \equiv C D_{\text {conv }}(\mathrm{NLTE})-C D_{\text {obs }}$.

In the above definition, the value of $C D_{\text {conv }}$ (NLTE) can be either computed explicitly from a model photosphere of the star, or estimated by using the interpolation relations given in Sect. 3.2.

Figure 8 shows the behavior of the index $R_{\mathrm{IRT}}(18542)$ versus $\log R_{\mathrm{HK}}^{\prime}$, for the subset of stars considered by Chmielewski (2000) with known $v \sin \hat{\imath}$. The $R_{\mathrm{IRT}}$ index has been calculated using the observed $C D$ values given by Chmielewski (2000). The $\log R_{\mathrm{HK}}^{\prime}$ values are also from Chmielewski (2000), except for HD 186408 and HD 186427, for which we adopted the values listed by García-López et al. (1993). The $v \sin \hat{\imath}$ values are either from Uesugi \& Fukuda (1970), or from Bernacca \& Perinotto (1970), except for HD 131977, for which the adopted value is from Marrese et al. (2003). In Fig. 8 we have added the data point corresponding to the Sun; we have also indicated with a different symbol the two stars with $\log g<4.0$ - outside the range considered by our grid, although by not much.

The data points shown in Fig. 8 display a scatter significantly higher than the errors introduced by the interpolation formulae of Sect. 3.2, as it can be expected from a non-homogeneous sample of observations. In particular, some of the effects that could add significant scatter to the data points could be: different instrumental resolutions (if less than $R \approx 50000$, this can be a factor); errors in stellar parameters (mainly $v \sin \hat{\imath}$ ); errors in $\log R_{\mathrm{HK}}^{\prime}$ measurements; errors in the measurements of $C D$ s due to the low $\mathrm{S} / \mathrm{N}$ in the line core; intrinsic variability (non-simultaneous observations), etc.

Nevertheless, a correlation can be clearly seen. More specifically: the linear correlation coefficient (Pearson's $r$ ) is: $r=0.73$. A strong correlation is also found with other, non-parametric correlation coefficients: Spearman's rank-order correlation, for instance, is $r_{\mathrm{s}}=0.77$. The significance of the latter coefficient - the probability of obtaining a higher value under the null hypothesis of uncorrelated quantities - is $1.5 \times 10^{-4}$.

A linear best fit gives:

$Y=(0.24 \pm 0.06) \times X+(1.4 \pm 0.3)$,

where $X=\log R_{\mathrm{HK}}^{\prime}$ and $Y=R_{\mathrm{IRT}}(\lambda 8542)$. The covariance of the two coefficients is 0.014 .

To facilitate a comparison with Chmielewski (2000), we have also fitted the data with a parabola with a minimum constrained at $\log R_{\mathrm{HK}}^{\prime}=-5.10$ :

$Y=(0.29 \pm 0.08) \times X^{\prime 2}+(0.17 \pm 0.02)$,

where $X^{\prime} \equiv X+5.10$, and the covariance of the fit coefficients is -0.0012 . In this case, the quadratic coefficient of Eq. (6) can be directly compared with the corresponding value given by Chmielewski (2000) (its absolute value, actually: in that paper the fitted quantity is the central line depth). Our estimated coefficient is about $60 \%$ of the value estimated by Chmielewski (2000); the reason is, of course, that we have removed the contribution of rotational broadening (correlated with $\log R_{\mathrm{HK}}^{\prime}$ ).

The star with the smallest $R_{\mathrm{IRT}}$ is $\beta \mathrm{Aql}$ (HD 188512), a star of very low activity, suggested by Henry et al. (1996) to be in a phase analogous to the historical solar Maunder Minimum. Its measured $C D$ is 0.14 , while $R_{\mathrm{IRT}}=0.02$ : therefore, according to our calculations, its Ca II IRT profiles are in fact nearly photospheric.

\section{Conclusions}

The Ca II IRT lines are strong features of the spectra of latetype stars which are becoming increasingly commonly observed in a variety of astrophysical contexts. However, the interpretation of the profiles of those lines normally requires an NLTE analysis, unless profile-integrated properties only, such as $E Q W \mathrm{~s}$, are to be considered. Nevertheless, even in the case of the $E Q W \mathrm{~s}$, we found that in main-sequence, solar-type stars, LTE is a good approximation only in stars with $[\mathrm{A} / \mathrm{H}]>-1.0$.

In the context of chromospheric activity studies, the Ca II IRT lines can be very useful diagnostics. For such studies, there is an even stronger need for proper NLTE analyses, since the presence of a chromosphere mostly affects the line core profile, which is essentially formed in the less dense regions of the star's atmosphere (even in absence of a chromosphere). On the other hand, the line cores, and in particular the line $C D$ s, are less dependent than $E Q W$ on the photospheric parameters $\left(T_{\mathrm{eff}}\right.$, $\log g,[\mathrm{~A} / \mathrm{H}])$. However, a residual dependence is still present, which must be taken into account for proper quantitative studies. The main subject of this study is in fact to present detailed NLTE calculations of Ca II IRT line profiles, and in particular of their $E Q W \mathrm{~s}$ and $C D$ s, for main-sequence, solar-type stars without a chromosphere. Such calculations can be used as the zero point ("theoretical templates") for empirical investigation of the chromospheric contribution to the Ca II IRT line profiles.

In this paper we also discuss the results of our calculations, and, among other things, we show that the effect of rotational 
broadening must also be taken into account if the $C D$ is to be used as a proxy for chromospheric activity. We therefore defined a new index, $R_{\mathrm{IRT}}$ : the observed $C D$ relative to the photospheric value (computed in NLTE and convolved for $v \sin \hat{\imath}$ ). We found that this purely chromospheric index promises to be a good probe of stellar activity, at least in main-sequence stars.

Acknowledgements. We wish to thank R. Aznar Cuadrado and M. Rodonò for their critical reading of the manuscript, as well as the anonymous referee, whose suggestions helped to improve this paper. This research has made use of the SIMBAD database, operated at CDS, Strasbourg, France. This work was started while one of the authors (I.B.) was at Osservatorio Astronomico di Capodimonte, for which she gratefully acknowledges support from the Italian Ministry for Scientific and Technological Research (MURST - now: MIUR), through CoFin 2000 funding.

\section{References}

Allard, F., \& Hauschildt, P. H. 1995, ApJ, 445, 433

Bernacca, P. L., \& Perinotto, M. 1970, Contr. Oss. Astrof. Padova in Asiago, 239, 1

Busà, I., Andretta, V., Gomez, M. T., \& Terranegra, L. 2001, A\&A, 373, 993

Carlsson, M. 1986, A Computer Program for Solving Multi-Level Non-LTE Radiative Transfer Problems in Moving or Static Atmospheres, Report No. 33, Uppsala Astronomical Observatory

Cenarro, A. J., Cardiel, N., Gorgas, J., et al. 2001a, MNRAS, 326, 959

Cenarro, A. J., Gorgas, J., Cardiel, N., et al. 2001b, MNRAS, 326, 981

Cenarro, A. J., Gorgas, J., Cardiel, N., Vazdekis, A., \& Peletier, R. F. 2002, MNRAS, 329, 863

Chmielewski, Y. 2000, A\&A, 353, 666

Dempsey, R. C., Bopp, B. W., Henry, G. W., \& Hall, D. S. 1993, ApJS, 86, 293

Drake, J. J. 1991, MNRAS, 251, 369
Erdelyi-Mendes, M., \& Barbuy, B. 1991, A\&A, 241, 176

Fontenla, J. M., Avrett, E. H., \& Loeser, R. 1993, ApJ, 406, 319

García-López, R. J., Rebolo, R., Beckman, J. E., \& McKeith, C. D. 1993, A\&A, 273, 482

Grevesse, N., \& Sauval, A. J. 1998, Space Sci. Rev., 85, 161

Henry, T. J., Soderblom, D. R., Donahue, R. A., \& Baliunas, S. L. 1996, AJ, 111, 439

Jørgensen, U. G., Carlsson, M., \& Johnson, H. R. 1992, A\&A, 254, 258

Kurucz, R. L., \& Bell, B. 1995, Atomic Line Data, Kurucz CD-ROM No. 23, Cambridge, Mass.: Smithsonian Astrophysical Observatory

Kurucz, R. L., Furenlid, I., Brault, J., \& Testerman, L. 1984, Solar flux atlas from 296 to 1300 nm, Tucson, Ariz.: National Solar Observatory

Linsky, J. L., Hunten, D. M., Sowell, R., Glackin, D. L., \& Kelch, W. L. 1979, ApJS, 41, 481

Mallik, S. V. 1994, A\&AS, 103, 279

Mallik, S. V. 1997, A\&AS, 124, 359

Mantegazza, L. 1992, A\&A, 265, 527

Marrese, P. M., Boschi, F., \& Munari, U. 2003, A\&A, 406, 995

Mihalas, D. 1978, Stellar Atmospheres, 2nd ed. (San Francisco: Freeman)

Montes, D., Fernández-Figueroa, M. J., De Castro, E., et al. 2000, A\&AS, 146, 103

Munari, U., \& Tomasella, L. 1999, A\&AS, 137, 521

Noyes, R. W., Hartmann, L. W., Baliunas, S. L., Duncan, D. K., \& Vaughan, A. H. 1984, ApJ, 279, 763

Smith, G., \& Drake, J. J. 1987, A\&A, 181, 103

Smith, G., \& Drake, J. J. 1990, A\&A, 231, 125

Soderblom, D. R., Stauffer, J. R., Hudon, J. D., \& Jones, B. F. 1993, ApJS, 85, 315

Uesugi, A., \& Fukuda, I. 1970, Catalogue of rotational velocities of the stars, Contributions from the Institute of Astrophysics and Kwasan Observatory, University of Kyoto (Kyoto: University, Kwasan Observatory, Institute of Astrophysics)

Vernazza, J. E., Avrett, E. H., \& Loeser, R. 1976, ApJS, 30, 1 